\title{
STRONG ALGEBRABILITY OF SETS OF SEQUENCES AND FUNCTIONS
}

\author{
ARTUR BARTOSZEWICZ AND SZYMON GŁA̧B
}

(Communicated by Thomas Schlumprecht)

\begin{abstract}
We introduce a notion of strong algebrability of subsets of linear algebras. Our main results are the following. The set of all sequences from $c_{0}$ which are not summable with any power is densely strongly $\mathfrak{c}$-algebrable. The set of all sequences in $l^{\infty}$ whose sets of limit points are homeomorphic to the Cantor set is comeager and strongly $\mathfrak{c}$-algebrable. The set of all nonmeasurable functions from $\mathbb{R}^{\mathbb{R}}$ is $2^{\mathfrak{c}}$-algebrable. These results complete several by other authors, within the modern context of lineability.
\end{abstract}

\section{INTRODUCTION}

Assume that $B$ is a linear algebra, that is, a linear space that is also an algebra. According to [4], a subset $E \subset B$ is called algebrable whenever $E \cup\{0\}$ contains an infinitely generated algebra. If $\kappa$ is a cardinal, $E$ is said to be $\kappa$-algebrable if $E \cup\{0\}$ contains an algebra generated by an algebraically independent set with cardinality $\kappa$. Hence algebrability equals $\omega$-algebrability, where $\omega$ denotes the cardinality of the set $\mathbb{N}$ of positive integers. In addition to algebrability, there exist other related properties such as lineability, dense-lineability and spaceability; see [3], [8], 9] and [14.

In this note we will focus on algebrability. This notion was considered by many authors. For instance Aron, Pérez-García and Seoane-Sepúlveda proved in [4] that the set of non-convergent Fourier series is algebrable. Aron and Seoane-Sepúlveda established in [5] algebrability of everywhere surjective functions on $\mathbb{C}$, which was strengthened in [2]. Other algebrability results can be found in [11] and [12]. Here we will discuss some strengthening of the notion of algebrability.

For a cardinal $\kappa$, we say that $A$ is a $\kappa$-generated free algebra if there exists a subset $X=\left\{x_{\alpha}: \alpha<\kappa\right\}$ of $A$ such that any function $f$ from $X$ to some algebra $A^{\prime}$ can be uniquely extended to a homomorphism from $A$ into $A^{\prime}$. Then $X$ is called a set of free generators of the algebra $A$. A subset $X=\left\{x_{\alpha}: \alpha<\kappa\right\}$ of a commutative algebra $B$ generates a free subalgebra $A$ if and only if for each polynomial $P$ and any $x_{\alpha_{1}}, x_{\alpha_{2}}, \ldots, x_{\alpha_{n}}$ we have $P\left(x_{\alpha_{1}}, x_{\alpha_{2}}, \ldots, x_{\alpha_{n}}\right)=0$ if and only if $P=0$.

This leads us to the following definition. We say that a subset $E$ of a commutative linear algebra $B$ is strongly $\kappa$-algebrable if there exists a $\kappa$-generated free algebra $A$ contained in $E \cup\{0\}$. If additionally, $B$ is equipped with some topological structure and $A$ is dense in $B$, then we say that $E$ is densely strongly $\kappa$-algebrable.

Received by the editors May 24, 2011 and, in revised form, July 19, 2011 and July 23, 2011.

2010 Mathematics Subject Classification. Primary 15A03; Secondary 28A20, 46J10.

Key words and phrases. Algebrability, non-summable sequences, non-measurable functions, Banach-Mazur game, accumulation points, Cantor sets. 
Note that $X=\left\{x_{\alpha}: \alpha<\kappa\right\} \subset E$ is a set of free generators of a free algebra $A \subset E$ if and only if the set $\tilde{X}$ of elements of the form $x_{\alpha_{1}}^{k_{1}} x_{\alpha_{2}}^{k_{2}} \cdots x_{\alpha_{n}}^{k_{n}}$ is linearly independent and all linear combinations of elements from $\tilde{X}$ are in $E \cup\{0\}$. (In particular, it follows that sets considered in [7] were strongly algebrable.) It is easy to see that free algebras have no divisors of zero.

In general, there are subsets of linear algebras which are algebrable but not strongly algebrable. Let $c_{00}$ be a subset of $c_{0}$ consisting of all sequences with real terms equal to zero from some place. Having members $x_{1}, \ldots, x_{n}$ of some linear algebra and a polynomial $P$, an element $P\left(x_{1}, \ldots, x_{n}\right)$ will be called an algebraic combination of $x_{1}, \ldots, x_{n}$.

Proposition 1. The set $c_{00}$ is algebrable in $c_{0}$ but is not strongly 1-algebrable.

Proof. Note that $c_{00}$ is a linear subalgebra of $c_{0}$ which can be generated by a linearly independent set of cardinality $\omega$, namely, by $\left\{e_{1}, e_{2}, \ldots\right\}$, where $e_{i}$ is a sequence consisting of 1 on the $i$-th coordinate and zeros on the remaining coordinates. Note also that $c_{00}$ cannot be generated by finitely many elements, since any linear (or algebraic) combination of finitely many elements from $c_{00}$ eventually equals zero.

Suppose that $c_{00}$ is strongly 1 -algebrable; i.e., there is a sequence $a \in c_{00}$ which is algebraically independent. In particular, this means that the collection $\left\{a, a^{2}, a^{3}, \ldots\right\}$ is linearly independent. Since $a \in c_{00}$, there is $n \in \mathbb{N}$ such that $a(k)=0$ for $k \geq n$. Moreover $a^{m}(k)=0$ for all $k \geq n$ and $m \in \mathbb{N}$. Note that the linear space generated by $a, a^{2}, a^{3}, \ldots$ is isomorphic to a linear subspace of $\mathbb{R}^{n}$ and the restriction vectors $a_{\uparrow n}, a_{\uparrow n}^{2}, a_{\uparrow n}^{3}, \ldots$ are linearly independent in $\mathbb{R}^{n}$, a contradiction.

The aim of this note is to prove strong $\mathfrak{c}-$ algebrability of some sets of sequences and functions, where $\mathfrak{c}$ denotes cardinality of the continuum. These are: the set of all sequences tending to zero which are not summable with any power, the set of non-measurable functions, and the set of bounded real sequences whose set of accumulation points is of measure zero and can be decomposed into a finite set and a set homeomorphic to the Cantor set. This last set happens to be co-meager.

\section{Algebrability in $c_{0}$ And $l^{\infty}$}

To prove Theorem 2 we will use the idea from the paper [7. Our theorem is related to [10, Theorem 1.3], from which the spaceability of $c_{0} \backslash \bigcup\left\{l^{p}: p \geq 1\right\}$ is extracted as a consequence. Recall that a subset $E$ of a topological vector space $X$ is called spaceable if $E \cup\{0\}$ contains a closed infinite-dimensional subspace of $X$.

Theorem 2. The set $c_{0} \backslash \bigcup\left\{l^{p}: p \geq 1\right\}$ is densely strongly $\mathfrak{c}$-algebrable in $c_{0}$.

Proof. Let $\left\{r_{\alpha}: \alpha<\mathfrak{c}\right\}$ be a linearly independent (with respect to the field $\mathbb{Q}$ ) subset of positive reals containing 1 . Let $A$ be a linear algebra generated by the set

$$
S=\left\{\left(\frac{1}{\ln ^{r_{\alpha}}(n+1)}\right)_{n=1}^{\infty}: \alpha<\mathfrak{c}\right\} .
$$

We will show that any non-trivial algebraic combination of elements from $S$ is either a null sequence or it is not summable with any power. To prove this, we need to show that for every matrix $\left(k_{i l}: i \leq m, l \leq j\right)$ of non-negative integers with nonzero and distinct rows and any $\beta_{1}, \ldots, \beta_{m} \in \mathbb{R}$ which do not vanish simultaneously, 
the series

$$
\sum_{n=1}^{\infty}\left(\beta_{1} \frac{1}{\ln ^{k_{11} r_{\alpha_{1}}+\ldots+k_{1 j} r_{\alpha_{j}}(n+1)}}+\ldots+\beta_{m} \frac{1}{\ln ^{k_{m 1} r_{\alpha_{1}}+\ldots+k_{m j} r_{\alpha_{j}}(n+1)}}\right)
$$

is not summable with any power. Note that the sequence

$$
\left(\frac{1}{\ln ^{k_{11} r_{\alpha_{1}}+\ldots+k_{1 j} r_{\alpha_{j}}(n+1)}}\right)_{n \in \mathbb{N}}
$$

tends to zero; i.e. it is in $c_{0}$. We need to prove that the series (11) is divergent. Since the set $\left\{r_{\alpha}: \alpha<\mathfrak{c}\right\}$ is linearly independent, the numbers $k_{11} r_{\alpha_{1}}+\ldots+k_{1 j} r_{\alpha_{j}}, \ldots$, $k_{m 1} r_{\alpha_{1}}+\ldots+k_{m j} r_{\alpha_{j}}$ are distinct. To simplify the notation, put $k_{i}:=k_{i 1} r_{\alpha_{1}}+\ldots+$ $k_{i j} r_{\alpha_{j}}$ for every $i=1, \ldots, m$. We may assume that $k_{1}<k_{2}<\ldots<k_{m}$ and $\beta_{1} \neq 0$. Since $\frac{\ln ^{k_{i}}(n+1)}{\ln ^{k_{1}}(n+1)} \rightarrow \infty(n \rightarrow \infty)$ for all $i=2, \ldots, m$, we obtain that there is an $N$ such that

$$
\left|\beta_{1} \frac{1}{\ln ^{k_{1}}(n+1)}+\beta_{2} \frac{1}{\ln ^{k_{2}}(n+1)}+\ldots+\beta_{m} \frac{1}{\ln ^{k_{m}}(n+1)}\right| \geq \frac{\left|\beta_{1}\right|}{2 \ln ^{k_{1}}(n+1)}
$$

for all $n \geq N$. Since $\left(\frac{1}{\ln ^{q}(n+1)}\right)_{n=1}^{\infty} \notin l^{p}$ for any $p \geq 1$ and $q>0$, the result follows.

Now, we prove that $A$ is dense in $c_{0}$. Consider the subalgebra $A_{1}$ of space $c$ (of all convergent sequences with the sup-norm), generated by two elements: $\left(\frac{1}{\ln (n+1)}\right)_{n=1}^{\infty}$ and $1=(1,1,1, \ldots)$. Let $A_{2}$ be a subalgebra of $A$ generated by $\left(\frac{1}{\ln (n+1)}\right)_{n=1}^{\infty}$. Note that $A_{1}=\left\{x+(a, a, a, \ldots): x \in A_{2}, a \in \mathbb{R}\right\}$. Equip the set of positive integers $\mathbb{N}$ with the discrete topology and let $\alpha \mathbb{N}$ be a one-point compactification of $\mathbb{N}$. Note that $c$ is isometrically isomorphic to the space $C(\alpha \mathbb{N})$ of all continuous real functions on $\alpha \mathbb{N}$ with the sup-norm. Since $\left(\frac{1}{\ln (n+1)}\right)_{n=1}^{\infty}$ is a strictly decreasing sequence of positive numbers tending to zero, then it separates the points of $\alpha \mathbb{N}$. Therefore by the Stone-Weierstrass theorem, the algebra $A_{1}$ is dense in $c$.

Take any $x \in c_{0}$ and $\varepsilon>0$. There is $y \in A_{1}$ with $\|x-y\|<\varepsilon / 2$. Note that $\left|\lim _{n} y(n)\right| \leq \varepsilon / 2$. Put $\hat{y}=\left(y(1)-\lim _{n} y(n), y(2)-\lim _{n} y(n), y(3)-\lim _{n} y(n), \ldots\right) \in$ $c_{0}$. Then $\|x-\hat{y}\|<\varepsilon$. Since $\hat{y} \in A_{2} \subset A$, we obtain that $A_{2}$, and therefore $A$, is dense in $c_{0}$.

In the sequel we will need the following observation. Let $S$ be a set defined in the proof of Theorem 2, Let $S=\left\{a_{\alpha}: \alpha<\mathfrak{c}\right\}$ and let $\left(n_{k}\right)$ be any increasing sequence of natural numbers. Then $S^{\prime}:=\left\{\left(a_{\alpha}\left(n_{k}\right)\right)_{k \in \mathbb{N}}: \alpha<\mathfrak{c}\right\}$ is also a set of free generators in $c_{0}$. This easily follows from (3) .

In [1] it was proved that the set $E$ of all sequences from $l^{\infty}$ which are not convergent is $\mathfrak{c}$-lineable; i.e. there is a linear $\mathfrak{c}$-dimensional subspace of $l^{\infty}$ for which every non-zero element is divergent. This part is devoted to some strengthening of this result to obtain a theorem on algebrability.

We say that a function $f: \mathbb{R} \rightarrow \mathbb{R}$ fulfils the (N) Luzin condition if the images of null sets are null (i.e. of Lebesque measure zero). Note that all polynomials satisfy the $(\mathrm{N})$ Luzin condition and are finite-to-one.

Lemma 3. Let $f: \mathbb{R} \rightarrow \mathbb{R}$ be a finite-to-one continuous function satisfying the $(N)$ Luzin condition. Then the image $f(C)$ of any compact perfect zero-dimensional set $C$ is also compact perfect and zero-dimensional. 
Proof. Let $x \in f(C), y \in f^{-1}(\{x\})$ and take $y_{n} \rightarrow y$ with $y_{n} \neq y$ and $y_{n} \in C$ for all $n$. Then the sequence $\left(f\left(y_{n}\right)\right)$ tends to $x$, and it contains a subsequence all of whose elements are distinct from $x$. Therefore $f(C)$ is dense-in-itself. Clearly it is compact and thus $f(C)$ is perfect. Since $f(C)$ is null, it is also zero-dimensional.

For a sequence $x \in \mathbb{R}^{\mathbb{N}}$ let $\operatorname{LIM}(x)$ stands for the set of all limit points of $x$, i.e. $\operatorname{LIM}(x)=\left\{y \in \mathbb{R}: x\left(n_{k}\right) \rightarrow y\right.$ for some $\left.\left(n_{k}\right)\right\}$. Clearly if $x \in l^{\infty}$, then $\operatorname{LIM}(x)$ is non-empty. Note that $x \in l^{\infty}$ is convergent if and only if $\operatorname{LIM}(x)$ is a singleton. In general, $\operatorname{LIM}(x)$ is a non-empty compact subset of $\mathbb{R}$. On the other hand if $C$ is a non-empty compact subset of the reals, then there is a decomposition of $C$ into a perfect set $P \subset C$ (which can be empty) and a countable set $D \subset C$ (this is the famous Cantor-Bendixson theorem). Now, taking $\left(x_{n}\right)$ such that each point of $D$ appears in $\left(x_{n}\right)$ infinitely many times and the set of remaining points of $\left(x_{n}\right)$ is dense in $P$, we obtain $\operatorname{LIM}(x)=C$. Let $E_{\text {Cantor }}=\left\{x \in l^{\infty}: \operatorname{LIM}(x)\right.$ is the union of a finite set and a set homeomorphic to the Cantor set $\}$.

Let $A_{1}, A_{2}, \ldots$ be a partition of the set of even numbers in $\mathbb{N}$ into infinite sets. Let $B_{i}=A_{i} \cup\{2 i-1\}$. Then clearly $B_{1}, B_{2}, \ldots$ is a partition of $\mathbb{N}$. Let $x \in l^{\infty}$ be the characteristic function of even numbers. Then $\operatorname{LIM}(x)=\{0,1\}$ and $\bigcup_{n=1}^{\infty} \operatorname{LIM}\left(x_{\uparrow B_{n}}\right)=$ $\{1\}$. Therefore one cannot have the equality $\operatorname{LIM}(x)=\operatorname{cl}\left(\bigcup_{n=1}^{\infty} \operatorname{LIM}\left(x_{\uparrow B_{n}}\right)\right)$ for any partition $B_{1}, B_{2}, \ldots$. However, we easily obtain the following.

Lemma 4. Assume that $\{x(n): n \in \mathbb{N}\} \subset \operatorname{cl}\left(\bigcup_{n=1}^{\infty} \operatorname{LIM}\left(x_{\uparrow B_{n}}\right)\right)$. Then $\operatorname{LIM}(x)=$ $\operatorname{cl}\left(\bigcup_{n=1}^{\infty} \operatorname{LIM}\left(x_{\uparrow B_{n}}\right)\right)$.

A family $\mathcal{F}$ of infinite subsets of $\mathbb{N}$ is called almost disjoint if $A \backslash B$ is finite for every $A, B \in \mathcal{F}$. It is well-known that there is an almost disjoint family of cardinality $\mathfrak{c}$. The classical Brouwer theorem states that a subset of $\mathbb{R}$ is homeomorphic to the Cantor set if and only if it is compact, dense-in-itself and zero-dimensional (cf. [15, Theorem 7.4]). Note also that a meager compact set is zero-dimensional.

Theorem 5. $E_{\text {Cantor }}$ is strongly $\mathfrak{c}$-algebrable.

Proof. Let $\left\{a_{\alpha}: \alpha<\mathfrak{c}\right\}$ be a set of free generators of the algebra defined in the proof of Theorem 2, Let $C$ be the Cantor set. Let $N_{1}, N_{2}, \ldots$ be a partition of $\mathbb{N}$ into infinitely many infinite sets. Let $\left\{F_{\alpha}^{i}: \alpha<\mathfrak{c}\right\}$ be an almost disjoint family of subsets of $N_{i}$. For $\alpha<\mathfrak{c}$ define $x_{\alpha}(n)=a_{\alpha}(i)$ if $n \in N_{i} \backslash F_{\alpha}^{i}$, and define $x_{\alpha}$ on $F_{\alpha}^{i}$ such that $\mathrm{cl}\left(\left\{x_{\alpha}(n): n \in F_{\alpha}^{i}\right\}\right)=a_{\alpha}(i)+C$. Now let $A$ be the algebra generated by $\left\{x_{\alpha}: \alpha<\mathfrak{c}\right\}$.

Fix $x \in A \backslash\{0\}$. Then there exist $\alpha_{1}<\alpha_{2}<\ldots<\alpha_{m}<\mathfrak{c}$ as well as a non-trivial polynomial $P$ of $m$ variables with non-zero real coefficients such that $x=P\left(x_{\alpha_{1}}, x_{\alpha_{2}}, \ldots, x_{\alpha_{m}}\right)$. Note that $P\left(a_{\alpha_{1}}(i), a_{\alpha_{2}}(i), \ldots, a_{\alpha_{m}}(i)\right) \neq 0$ for all but finitely many $i \in \mathbb{N}$, and

$$
\lim _{i \rightarrow \infty} P\left(a_{\alpha_{1}}(i), a_{\alpha_{2}}(i), \ldots, a_{\alpha_{m}}(i)\right)=0 .
$$

Fix $i \in \mathbb{N}$ and $j \in\{1, \ldots, m\}$. Since $\left\{F_{\alpha}^{i}: \alpha<\mathfrak{c}\right\}$ is almost disjoint, there is $M \in \mathbb{N}$ such that $\left(F_{\alpha_{j}}^{i} \backslash\{1, \ldots, M\}\right) \cap \bigcup_{k \neq j} F_{\alpha_{k}}^{i}=\emptyset$. Hence for each $k \neq j$ we have $x_{\alpha_{k}}(n)=a_{\alpha_{k}}(i)$ for any $n \in F_{\alpha_{j}}^{i} \backslash\{1, \ldots, M\}$. Moreover $\operatorname{LIM}\left(\left(x_{\alpha_{j}}\right)_{\left\lceil F_{\alpha_{j}}^{i} \backslash\{1, \ldots, M\}\right.}\right)=$ $a_{\alpha_{j}}(i)+C$. If $n \in N_{i} \backslash \bigcup_{k=1}^{m} F_{\alpha_{k}}^{i}$, then $x_{\alpha_{k}}(n)=a_{\alpha_{k}}(i)$. Consequently

$$
\operatorname{LIM}\left(x_{\uparrow F_{\alpha_{j}}^{i} \backslash\{1, \ldots, M\}}\right)=\operatorname{LIM}\left(x_{\left\lceil F_{\alpha_{j}}^{i}\right.}\right)=C_{i j},
$$


where

$$
C_{i j}=P\left(\left\{a_{\alpha_{1}}(i)\right\} \times \ldots \times\left\{a_{\alpha_{j-1}}(i)\right\} \times\left(a_{\alpha_{j}}(i)+C\right) \times\left\{a_{\alpha_{j+1}}(i)\right\} \times \ldots \times\left\{a_{\alpha_{m}}(i)\right\}\right) .
$$

Therefore by Lemma 4 we have

$$
\operatorname{LIM}(x)=\mathrm{cl}\left(\bigcup_{i=1}^{\infty} \bigcup_{j=1}^{m} C_{i j}\right) .
$$

Now, by Lemma 3 the set $C_{i j}$ is homeomorphic to the Cantor set (and therefore of measure zero) if and only if the function $f_{i j}$ defined by the formula $f_{i j}=$ $P\left(a_{\alpha_{1}}(i), \ldots, a_{\alpha_{j-1}}(i), t, a_{\alpha_{j+1}}(i), \ldots, a_{\alpha_{m}}(i)\right)$ is not constant. If $f_{i j}$ is constant, then $C_{i j}=\left\{P\left(a_{\alpha_{1}}(i), \ldots, a_{\alpha_{m}}(i)\right)\right\}$ is a singleton.

Claim. For all but finitely many $i$ 's there exists $j$ such that $C_{i j}$ is homeomorphic to the Cantor set.

Proof of Claim. Suppose to the contrary that there are infinitely many $i_{1}<i_{2}<\ldots$ such that for each $j$ every function $f_{i_{k} j}$ is constant. By the observation we did after Theorem 2, $S^{\prime}=\left\{\left(a_{\alpha}\left(i_{k}\right)\right)_{k \in \mathbb{N}}: \alpha<\mathfrak{c}\right\}$ is a set of free generators. Note that the derivative $f_{i_{k} j}^{\prime}$ equals zero. Since each partial derivative $P_{j}$ of polynomial $P$ is also a polynomial and $S^{\prime}$ forms a set of free generators, then $P_{j}$ equals zero. Therefore $P$ is constant. But $P$ does not have a constant term. Hence $P$ equals zero, which is a contradiction. This proves the Claim.

The closure of a null set is not necessarily null. Hence we need to specify what exactly we add in the closure of the set on the right side of equation (4). Take any convergent sequence $\left(x_{n}\right)$ from

$$
\bigcup_{i=1}^{\infty} \bigcup_{j=1}^{m} C_{i j}
$$

Let $x_{0}=\lim _{n} x_{n}$. Without loss of generality we may assume that all elements of the sequence are in $\bigcup_{i=1}^{\infty} C_{i 1}$. If there is $n_{0}$ such that all elements of the sequence are in $\bigcup_{i=1}^{n_{0}} C_{i 1}$, so is $x_{0}$. Therefore we may assume that there is an increasing sequence of indices $\left(k_{n}\right)$ with

$$
x_{n} \in P\left(\left(a_{\alpha_{1}}\left(k_{n}\right)+C\right) \times\left\{a_{\alpha_{2}}\left(k_{n}\right)\right\} \times \ldots \times\left\{a_{\alpha_{m}}\left(k_{n}\right)\right\}\right) .
$$

Thus there is a sequence $\left(y_{n}\right)$ of elements of $C$ such that

$$
x_{n}=P\left(a_{\alpha_{1}}\left(k_{n}\right)+y_{n}, a_{\alpha_{2}}\left(k_{n}\right), \ldots, a_{\alpha_{m}}\left(k_{n}\right)\right) .
$$

Since $C$ is compact, we may assume that $y_{n}$ tends to some $y \in C$. Since $a_{\alpha_{i}} \in c_{0}$, then

$$
x_{0}=P(y, 0, \ldots, 0) \in P(C \times\{(0, \ldots, 0)\}) .
$$

Hence

$$
\operatorname{cl}\left(\bigcup_{i=1}^{\infty} \bigcup_{j=1}^{m} C_{i j}\right)=\bigcup_{i=0}^{\infty} \bigcup_{j=1}^{m} C_{i j}
$$

where we put $a_{\alpha_{i}}(0)=0$. Thus by (41) the set of $\operatorname{limit}$ points $\operatorname{LIM}(x)$ is a countable union of null sets. Since it is compact, it is also zero-dimensional. Note that for fixed $i$ the intersection $C_{i 1} \cap \ldots \cap C_{i m}$ is non-empty; namely, it contains $P\left(a_{\alpha_{1}}(i), \ldots, a_{\alpha_{m}}(i)\right)$. Therefore by the Claim, all but finitely many sets of the form $\bigcup_{j=1}^{m} C_{i j}$ are homeomorphic to the Cantor set. Therefore $\operatorname{LIM}(x)$ is the union of a finite set and a countable union of sets homeomorphic to the Cantor set. If we 
remove from $\operatorname{LIM}(x)$ all its isolated points (there are finitely many of them), then the remaining part is the union of dense-in-itself sets, and therefore, as a dense-initself, zero-dimensional and compact set, it is homeomorphic to the Cantor set. To sum up, $x \in E_{\text {Cantor, }}$, as required.

By $B(x, \varepsilon)$ we denote the closed ball with the radius $\varepsilon>0$ centred at the point $x$. For $A \subset l^{\infty}$ put $\operatorname{LIM}(A)=\bigcup\{\operatorname{LIM}(x): x \in A\}$.

Lemma 6. Let $B_{1} \supset B_{2} \supset B_{3} \supset \ldots$ be a sequence of closed balls in $l^{\infty}$ such that the corresponding sequence of their radii tends to zero. Then $\operatorname{LIM}\left(\bigcap_{n} B_{n}\right)=$ $\bigcap_{n} \operatorname{LIM}\left(B_{n}\right)$.

Proof. The inclusion $\subset$ is trivial. As for the converse, suppose that $a \in \bigcap_{n} \operatorname{LIM}\left(B_{n}\right)$. There is $x_{n} \in B_{n}$ and $m_{1}^{n}<m_{2}^{n}<\ldots$ with $\lim _{k} x_{n}\left(m_{k}^{n}\right)=a$. Since the sequence of diameters of the $B_{n}$ 's tends to zero, $\left(x_{n}\right)$ is a Cauchy sequence in $l^{\infty}$. Let $x=\lim _{n} x_{n}$. We will show that $a \in \operatorname{LIM}(x) \subset \operatorname{LIM}\left(\bigcap_{n} B_{n}\right)$.

Suppose to the contrary that there is $\varepsilon>0$ and a positive integer $m$ such that $|x(k)-a| \geq \varepsilon$ for any $k \geq m$. By hypothesis, there is $n_{0}$ such that $\operatorname{diam}\left(B_{n}\right)<\varepsilon / 2$ for $n \geq n_{0}$. But $a \in \operatorname{LIM}\left(x_{n}\right)$ for any $n$; therefore for fixed $n \geq n_{0}$ we have $\left|x_{n}(k)-a\right|<\varepsilon / 2$ for infinitely many $k$ 's. Hence for each fixed $n \geq n_{0}$ there are infinitely many $k$ with

$$
\left|x_{n}(k)-x(k)\right| \geq|x(k)-a|-\left|x_{n}(k)-a\right| \geq \varepsilon-\varepsilon / 2=\varepsilon / 2 .
$$

But this contradicts the fact that $x_{n}$ tends to $x$ in $l^{\infty}$.

The Banach-Mazur game is an infinite topological game. Fix a topological space $X$ and a subset $A$ of it. Two players play open sets: player I starts with some non-empty open subset $U_{1}$, player II responds with an open subset $V_{1}$ of $U_{1}$, then player I takes some open set $U_{2} \subset V_{1}$, and player II responds with an open subset $V_{2}$ of $U_{2}$, etc. The second player wins if $\emptyset \neq \bigcap_{n} V_{n} \subset A$. The Banach-Mazur theorem states that player II has a winning strategy in this game if and only if $A$ is co-meager in $X$; cf. [15, Theorem 8.33] and [16, Chap. 6].

Let $E_{\text {finite }}=\left\{x \in l^{\infty}:\{x(n): n \in \mathbb{N}\}\right.$ is finite $\}$. It is clear that $E_{\text {finite }}$ is a dense $\mathfrak{c}$-generated algebra.

Theorem 7. The set $E_{\text {Cantor }}$ is co-meager in $l^{\infty}$.

Proof. To prove the co-meagerness of $E_{\text {Cantor }}$ we will apply the Banach-Mazur game. We need to define a winning strategy for player II.

Player I plays an arbitrary non-empty open set $U_{1} \subset l^{\infty}$. There is $x_{1} \in l^{\infty}$ and $\varepsilon>0$ with $B\left(x_{1}, \varepsilon\right) \subset U_{1}$. Observe that we may assume that $x_{1} \in E_{\text {finite }}$ and $\operatorname{LIM}\left(x_{1}\right)$ is not a singleton.

Let $\operatorname{LIM}\left(x_{1}\right)=\left\{a_{1,1}<a_{1,2}<\ldots<a_{1, n_{1}}\right\}$. Let $\varepsilon_{1}<\frac{1}{4} \min \left\{a_{1, i}-a_{1, i-1}: i=\right.$ $\left.2, \ldots, n_{1}\right\}$ and $0<\varepsilon_{1}<\varepsilon / 2$. Then

$$
B\left(x_{1}, \varepsilon_{1}\right) \subset B\left(x_{1}, \varepsilon\right) \subset U_{1} .
$$

Note that $\operatorname{LIM}\left(B\left(x_{1}, \varepsilon_{1}\right)\right)$ equals the disjoint union of intervals $\left[a_{1, i}-\varepsilon_{1}, a_{1, i}+\varepsilon_{1}\right]$. Player II plays $V_{1}:=B\left(x_{1}, \varepsilon_{1}\right) \subset U_{1}$. Additionally define $N_{1, i}=\left\{n: x_{1}(n)=a_{1, i}\right\}$.

Player I plays an arbitrary non-empty open set $U_{2} \subset V_{1}$. There is $x_{2} \in l^{\infty}$ and $\varepsilon>0$ with $B\left(x_{2}, \varepsilon\right) \subset U_{2}$. We may assume that $x_{2} \in E_{\text {finite }}$ and that each $\operatorname{LIM}\left(\left(x_{2}\right) \uparrow N_{1, i}\right)$ is not a singleton $\left(i=1, \ldots, n_{1}\right)$. 
Let $\operatorname{LIM}\left(x_{2}\right)=\left\{a_{2,1}<a_{2,2}<\ldots<a_{2, n_{2}}\right\}$. Let $\varepsilon_{2}<\frac{1}{4} \min \left\{a_{2, i}-a_{2, i-1}: i=\right.$ $\left.2, \ldots, n_{2}\right\}$ and $0<\varepsilon_{2}<\varepsilon_{1} / 2$. Then

$$
B\left(x_{2}, \varepsilon_{2}\right) \subset B\left(x_{2}, \varepsilon\right) \subset U_{2} .
$$

Note that $\operatorname{LIM}\left(B\left(x_{2}, \varepsilon_{2}\right)\right)$ equals the disjoint union of intervals $\left[a_{2, i}-\varepsilon_{2}, a_{2, i}+\varepsilon_{2}\right]$. Player II plays $V_{2}:=B\left(x_{2}, \varepsilon_{2}\right) \subset U_{2}$. Additionally define $N_{2, i}=\left\{n: x_{2}(n)=a_{2, i}\right\}$, etc.

Note that the closure of $V_{n+1}$ is contained in $V_{n}$. Since $l^{\infty}$ is complete and $\varepsilon_{n} \rightarrow 0, \bigcap_{n} V_{n}$ is non-empty and, in fact, it reduces to a point, say $x_{0}$. Moreover in every step $n$ of the game each subinterval of $\operatorname{LIM}\left(V_{n}\right)$ is split into at least two but finitely many subintervals of $\operatorname{LIM}\left(V_{n+1}\right)$ and the set $\operatorname{LIM}\left(V_{n+1}\right)$ is a union of finitely many compact intervals. Hence $\bigcap_{n} \operatorname{LIM}\left(V_{n}\right)$ is homeomorphic to the Cantor set. By Lemma 6 the set $\operatorname{LIM}\left(\bigcap_{n} V_{n}\right)=\operatorname{LIM}\left(x_{0}\right)$ is homeomorphic to the Cantor set, and therefore $E_{\text {Cantor }}$ is a co-meager subset of $l^{\infty}$.

The above results lead us to the following natural questions.

Problem 8. $\quad$ 1. Is $E_{\text {Cantor }}$ densely algebrable in $l^{\infty}$ ?

2. Does $E_{\text {Cantor }} \cup\{0\}$ contain a co-meager algebra?

3. Is the set $\left\{x \in l^{\infty}: \operatorname{LIM}(x)\right.$ is homeomorphic to the Cantor set $\} \mathfrak{c}$-algebrable?

At the end of this section we will show an example of a spaceable set in $l^{\infty}$, which is even not 1-algebrable. By $E \subset l^{\infty}$ denote the set of all sequences which do not attain their supremum. Gurariy and Quarta have shown in [14 that $E$ is spaceable.

Theorem 9. E is not 1-algebrable.

Proof. Suppose to the contrary that $E \cup\{0\}$ contains a non-trivial algebra. Then there is $x \in E$ such that any linear combination of $x, x^{2}, x^{3}, \ldots$ is either in $E$ or equals 0 . Note that $x^{2} \in E$. Let $a=\sup _{n} x^{2}(n)>0$. Then $\sup _{n} a^{-1} x^{2}(n)=1$ and $\inf _{n} a^{-1} x^{2}(n) \geq 0$. Consider the following polynomial of one variable:

$$
P(t)=2 t^{3}-3 a^{-1} x^{2}(1) t^{2} .
$$

It is easy to see that $P$ attains its supremum on the unit interval $[0,1]$ at the point $a^{-1} x^{2}(1)$. Therefore the sequence $P\left(a^{-1} x^{2}\right)$ attains its supremum at $P\left(a^{-1} x^{2}\right)(1)$.

\section{NON-MEASURABle FUnCTIONS}

The simplest way to get a non-measurable function is to take a characteristic function of a non-measurable set. The most common way to get a non-measurable set is to use the Vitali or the Bernstein constructions. A set $B \subset \mathbb{R}$ is called a Bernstein set provided that $B \cap P \neq \emptyset$ and $B^{c} \cap P \neq \emptyset$ for any perfect subset $P$ of the real line.

Suppose that $\left\{B_{\alpha}<\mathfrak{c}\right\}$ is a decomposition of $\mathbb{R}$ into $\mathfrak{c}$ pairwise disjoint Bernstein sets. Let $S \varsubsetneqq \mathfrak{c}$. Then $B:=\bigcup_{\alpha \in S} B_{\alpha}$ is also a Bernstein set. To see this, fix $\alpha \in S$ and $\beta \in \mathfrak{c} \backslash S$. Then $B_{\alpha}$ and $B_{\beta}$ have non-empty intersections with every perfect set $P$, and consequently their supersets $B$ and $B^{c}$, respectively, also satisfy the same condition. Using this observation and [13, Theorem 2.2] we obtain that the set of all non-measurable functions in $\mathbb{R}^{\mathbb{R}}$ is $2^{\mathfrak{c}}$-lineable. Note that there exists a function $F: \mathbb{R} \rightarrow \mathbb{R}$ such that $F^{-1}(\{x\})$ is a Bernstein set for every $x \in \mathbb{R}$. 
For any set $X$ we can consider $X^{\mathbb{R}}$ as a linear algebra under pointwise addition and product. We say that a family $\left\{A_{s}: s \in S\right\}$ of subsets of $X$ is independent if for any distinct $s_{1}, s_{2}, \ldots, s_{n} \in S$ and any $\varepsilon_{i} \in\{-1,1\}$ for $i=1, \ldots, n$ the set $A_{s_{1}}^{\varepsilon_{1}} \cap \ldots \cap A_{s_{n}}^{\varepsilon_{n}}$ is non-empty, where $B^{1}=B$ and $B^{-1}=X \backslash B$ for any $B \subset X$. Note that for any set of cardinality $\kappa$ there is an independent family of cardinality $2^{\kappa}$ (see [6]).

Theorem 10. Let $X$ be a set of cardinality $\kappa$. Then the set of all functions from $\mathbb{R}^{X}$ which are not constant is $2^{\kappa}$-algebrable.

Proof. We may assume that $X$ is of the form $Y \times \mathbb{N}$ with $Y$ of cardinality $\kappa$. Let $\left\{A_{\alpha}: \alpha<2^{\kappa}\right\}$ be an independent family of subsets of $Y$. For any $\alpha<2^{\kappa}$ define $f_{\alpha}: Y \times \mathbb{N} \rightarrow \mathbb{R}$ in the following way. If $x \notin A_{\alpha}$, put $f_{\alpha}(x, n)=0$ for any $n \in \mathbb{N}$. If $x \in A_{\alpha}$, put $f_{\alpha}(x, n)=\frac{1}{\ln (n+1)}$. We will show that $\left\{f_{\alpha}: \alpha<2^{\kappa}\right\}$ generate an algebra of non-constant functions.

Take any $\alpha_{1}<\alpha_{2}<\ldots<\alpha_{n}<2^{\kappa}$ and any non-zero polynomial $P$ without a free term. Consider a function $f:=P\left(f_{\alpha_{1}}, \ldots, f_{\alpha_{n}}\right)$. Note that $f$ on $\left(A_{\alpha_{1}}^{1} \cap A_{\alpha_{2}}^{-1}\right.$ $\left.\cap \ldots \cap A_{\alpha_{n}}^{-1}\right) \times \mathbb{N}$ is equal to $P\left(f_{\alpha_{1}}, 0, \ldots, 0\right)$. Hence $f$ is non-constant or equal to zero on the non-empty set $\left(A_{\alpha_{1}}^{1} \cap A_{\alpha_{2}}^{-1} \cap \ldots \cap A_{\alpha_{n}}^{-1}\right) \times \mathbb{N}$. Similarly for other sets of the

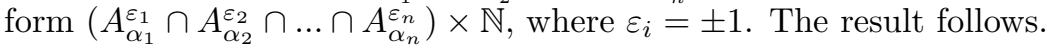

Finally, by $E \subset \mathbb{R}^{\mathbb{R}}$ we denote the set of all functions $f: \mathbb{R} \rightarrow \mathbb{R}$ such that $f^{-1}(\{x\})$ is either an empty set or a Bernstein set for any $x \in \mathbb{R}$. Note that any function from $E$ is Lebesgue non-measurable and does not have the Baire property.

Theorem 11. The set $E$ is $2^{\mathfrak{c}}$-algebrable.

Proof. Let $F: \mathbb{R} \rightarrow \mathbb{R}$ be a function such that $F^{-1}(\{t\})$ is a Bernstein set for any $t \in \mathbb{R}$. Let $\left\{f_{\alpha}: \alpha<2^{\mathfrak{c}}\right\}$ be a set of free generators in $\mathbb{R}^{X}$ given in Theorem 10, The family $\left\{f_{\alpha} \circ F: \alpha<2^{\mathfrak{c}}\right\}$ generates a free algebra contained in $E \cup\{0\}$.

\section{ACKNOWLEDGEMENTS}

The authors are indebted to the referee for suggesting several references and for helpful comments and suggestions that improved the text. The authors have been supported by the Polish Ministry of Science and Higher Education Grant No. N N201 414939 (2010-2013).

\section{REFERENCES}

[1] A. Aizpuru, C. Pérez-Eslava and J. B. Seoane-Sepúlveda, Linear structure of sets of divergent sequences and series, Linear Algebra Appl. 418 (2006), no. 2-3, 595-598. MR2260214 (2008h:40001)

[2] R. M. Aron, J. A. Conejero, A. Peris and J. B. Seoane-Sepúlveda, Uncountably generated algebras of everywhere surjective functions, Bull. Belg. Math. Soc. Simon Stevin 17 (2010), 571-575. MR2731374 (2011g:46041)

[3] R. Aron, V. I. Gurariy and J. B. Seoane-Sepúlveda, Lineability and spaceability of sets of functions on $\mathbb{R}$, Proc. Amer. Math. Soc. 133 (2005), no. 3, 795-803. MR2113929 (2006i:26004)

[4] R. M. Aron, D. Pérez-García and J. B. Seoane-Sepúlveda, Algebrability of the set of nonconvergent Fourier series, Studia Math. 175 (2006), no. 1, 83-90. MR2261701(2007k:42007)

[5] R. M. Aron and J. B. Seoane-Sepúlveda, Algebrability of the set of everywhere surjective functions on $\mathbb{C}$, Bull. Belg. Math. Soc. Simon Stevin 14 (2007), no. 1, 25-31. MR2327324 (2008d:26016)

[6] B. Balcar and F. Franěk, Independent families in complete Boolean algebras, Trans. Amer. Math. Soc. 274 (1982), no. 2, 607-618. MR675069 (83m:06020) 
[7] A. Bartoszewicz, S. Głąb and T. Poreda, On algebrability of nonabsolutely convergent series, Linear Algebra Appl. 435 (2011), no. 5, 1025-1028. MR2807216

[8] F. Bayart, Topological and algebraic genericity of divergence and universality, Studia. Math. 167 (2005), 161-181. MR2134382 (2006b:46024)

[9] L. Bernal-González, Dense-lineability in spaces of continuous functions, Proc. Amer. Math. Soc. 136 (2008), 3163-3169. MR2407080 (2009c:46038)

[10] G. Botelho, D. Diniz, V. V. Favaro and D. Pellegrino, Spaceability in Banach and quasiBanach sequence spaces, Linear Algebra Appl. 434 (2011), 1255-1260. MR2763584

[11] F. J. García-Pacheco, M. Martín and J. B. Seoane-Sepúlveda, Lineability, spaceability, and algebrability of certain subsets of function spaces, Taiwanese J. Math. 13 (2009), no. 4, 12571269. MR2543741(2010h:46072)

[12] F. J. García-Pacheco, N. Palmberg and J. B. Seoane-Sepúlveda, Lineability and algebrability of pathological phenomena in analysis, J. Math. Anal. Appl. 326 (2007), no. 2, 929-939. MR.2280953 (2007i:26003)

[13] F. J. García-Pacheco and J. B. Seoane-Sepúlveda, Vector spaces of non-measurable functions, Acta Math. Sin. (Engl. Ser.) 22 (2006), no. 6, 1805-1808. MR2262440 (2007i:28006)

[14] V. I. Gurariy and L. Quarta, On lineability of sets of continuous functions, J. Math. Anal. Appl. 294 (2004), no. 1, 62-72. MR2059788 (2005c:46026)

[15] A. S. Kechris, Classical descriptive set theory, Graduate Texts in Mathematics, 156. SpringerVerlag, New York, 1995. MR1321597 (96e:03057)

[16] J.C. Oxtoby, Measure and Category, Springer-Verlag, New York, 1980. MR.584443 (81j:28003)

Institute of Mathematics, Technical University of Łódź, Wólczańska 215, 93-005 ŁÓDŹ, POLAND

E-mail address: arturbar@p.lodz.pl

Institute of Mathematics, Technical University of Łódź, Wólczańska 215, 93-005 ŁÓDŹ, POLAND

E-mail address: szymon.glab@p.lodz.pl 